\title{
Transcriptome profiling of 6-OHDA model of Parkinson's disease
}

\author{
Maria I. Shadrina ${ }^{1}$, Elena V. Filatova ${ }^{1}$, Anelya Kh. Alieva ${ }^{1}$, Alla V. Stavrovskaya ${ }^{2}$, \\ Rudolph M. Khudoerkov ${ }^{2}$, Svetlana A. Limborska ${ }^{1}$, Sergei N. Illarioshkin ${ }^{2}$, Petr A. Slominsky ${ }^{1}$ \\ ${ }^{1}$ Institute of Molecular Genetics, Russian Academy of Sciences, Moscow, Russia \\ ${ }^{2}$ Scientific Centre of Neurology, Russian Academy of Medical Sciences, Moscow, Russia \\ Email: FilatovaEV@,img.ras.ru
}

Received 18 April 2013; revised 22 May 2013; accepted 30 May 2013

Copyright (C) 2013 Maria I. Shadrina et al. This is an open access article distributed under the Creative Commons Attribution License, which permits unrestricted use, distribution, and reproduction in any medium, provided the original work is properly cited.

\begin{abstract}
Analysis of monogenic forms and candidate genes of Parkinson's disease (PD) does not allow to describe completely the contribution of genetic factors to the etiopathogenesis of the disorder. An approach associated with an analysis of changes in a transcriptome pattern during the development of the disease in model objects can be used to identify new candidate genes that are involved in the pathogenesis of PD. In this work, we performed a transcriptome analysis of a PD model, created via stereotaxic unilateral introduction of the 6-hydroxidopamine (6-OHDA) into the substantia nigra pars compacta (SNpc) of a rat brain, to identify new candidate genes for PD. We studied transcriptome alterations in the substantia nigra of the rat brains 2 weeks after toxin administration, when the rats developed the Parkinson-like phenotype, and 4 weeks after toxin administration, when maximal changes in the behavior of animals were observed. The transcriptome analysis of the substantia nigra of the rat brains at the first time point (2 weeks) revealed changes in expression of genes that were clustered with high significance $(p<0.01$, modified Fisher extract $p$ value) into three metabolic pathways according to protein participation: modification of the extracellular matrix, signal transduction (including genes encoding signal peptides), and inflammation processes. This likely indicates that, during this time nonspecific effects associated with the response to surgery took place in the substantia nigra of the rats. Concomitantly, the situation changed dramatically and a response associated with damage to the nervous tissue was observed 4 weeks after neurotoxin administration. As a result, we identified five metabolic pathways containing predominantly genes, that encode protein products that are involved in the
\end{abstract}

processes of neuron projection, normal functioning of the soma and dendrites of neurons, synaptic transmission, and transmission of nerve impulses $(p<0.01$, modified Fisher extract $p$ value).

Keywords: 6-OHDA; Parkinson's Disease; Transcriptome

\section{INTRODUCTION}

Parkinson's disease (PD) is one of the major neurodegenerative disorders that are characterized by progressive degeneration of dopaminergic neurons in the substantia nigra pars compacta $(\mathrm{SNpc})$ region of the brain which leads to massive loss of dopamine (DA) in the caudate nucleus and putamen [1]. Mutations in genes such as SNCA, PARK2, PINK1, PARK7 (DJ-1), and LRRK2 have been reported to lead to the development of monogenic forms of PD. Moreover, several candidate genes with an unclear role in the pathogenesis of PD have been identified [2,3]. However, the analysis of these genes does not allow the complete description of the contribution of genetic factors to the etiopathogenesis of the disorder. An approach associated with the analysis of changes in the transcriptome pattern during the development of the disease in model objects can be used to identify new candidate genes that are involved in the pathogenesis of PD. One of the most widespread methods of PD modeling is the administration of a neurotoxin to rodents. This technique allows the creation of models that reproduce well the pathological and behavioral changes that are specific to PD [4]. The 6-hydroxidopamine (6-OHDA) model, which is prototypically based on the local injection of the neurotoxin, was the first animal model of PD ever generated [5]. 6-OHDA is a hydroxylated analogue of DA with high affinity to DAT, which 
transports the toxin inside dopaminergic neurons. 6OHDA cannot pass through the blood-brain barrier; thus, it is introduced either directly into the nigrostriatal pathway, striatum, SNpc (usually unilaterally), the lateral ventricle of the brain, or the big occipital cistern [6,7]. 6-OHDA causes massive anterograde degeneration of the nigrostriatal pathway after the injection into the SNpc. $\mathrm{SNpc}$ neurons begin to die within the first $12 \mathrm{~h}$ postinjection, whereas marked lesion of striatal dopaminergic terminals, paralleled by DA depletion, is established within 2 - 3 days. This procedure provides the highest level of nigral cell loss and striatal DA depletion obtainable in PD animal models (90\% - 100\%) [4]. The mechanism of action of 6-OHDA is substantially related to its prooxidative properties. 6-OHDA accumulates in the cytosol and undergoes prompt autooxidation, thus promoting a high rate of hydrogen peroxide formation. As an additional mechanism, 6-OHDA can accumulate in mitochondria, where it inhibits complex I activity. The lesion obtained using 6-OHDA is highly reproducible, which represents a considerable added value when new therapeutic strategies are to be investigated and clear neuroprotective effects must be demonstrated [4].

In this work, we performed a transcriptome analysis of a PD model created via stereotaxic unilateral introduction of 6-OHDA into the SNpc of the rat brain, to identify new candidate genes for PD.

\section{MATERIALS AND METHODS}

\subsection{Study Design}

Male Wistar rats (weight, $250 \pm 30 \mathrm{~g}$ ) were used in this study. The rats were kept in a vivarium with an environmental temperature of $22^{\circ} \mathrm{C}$, a 12 -h light/dark cycle (7:00 am onset) and free access to water and food. The experiments were carried out in strict accordance with IACUC guidelines. All rats were divided into four groups (10 animals in each group): two experimental and two control groups. The DA neuron-selective neurotoxin 6-OHDA hydro-bromide in $0.1 \%$ L-ascorbic acid $0.9 \%$ saline was administered using a Hamilton syringe with a 27-gauge needle to obtain a unilateral nigrostriatal lesion. The rats were anesthetized with $50 \mathrm{mg} / \mathrm{kg}$ of ketamine and $5 \mathrm{mg} / \mathrm{kg}$ of benzodiazepine intraperitoneally (i.p.). A burr hole was made in the skull using a dental drill, and after careful piercing of the dura mater, the needle of the Hamilton syringe was inserted vertically according to the stereotaxic coordinates: $-4.8 \mathrm{~mm}$ posterior and $2.0 \mathrm{~mm}$ lateral to bregma, $-7.0 \mathrm{~mm}$ with reference to the dura. Three microliters of the 6-OHDA solution was infused at a rate of $0.4 \mu \mathrm{L} / \mathrm{min}$ for a total infusion time of $7.5 \mathrm{~min}$. Sham-operated rats (control groups) were infused with an equivalent amount of vehicle. The needle was left in place for $1.5 \mathrm{~min}$ after the delivery of the reagent and then slowly retracted.

\subsection{Behavioral Study}

Testing of the motor activity of rats was carried out using the "open field" method for $3 \mathrm{~min}$. The number of crossed squares and number of racks were taken into account. The rats were then placed into the elevated $\mathrm{X}$-labyrinth, where the behavior of the animals was estimated for 3 min based on the following parameters: the preference for open arm (OA) or closed arm (CA) at the beginning of the experiment; the latent period of entry; and the time of stay in the OA or the CA. Registration of the behavior of animals was carried out using a web camera. The analysis of the rotational behavior of rats after subcutaneous injection of apomorphine was carried out to test the unilateral damage of DA neurons.

\subsection{Immunohistochemical Study}

The rat brains were analyzed 4 weeks after injection of the toxin, to estimate the loss of DA neurons and the decrease in dopamine synthesis caused by the introduction of 6-OHDA. The preparation of the brain slices for immunohistochemical study was carried out in accordance with the method described in the work of Chen and colleagues [8]. Quantitative evaluation of tyrosine hydroxyllase was performed using polyclonal antibodies (Sigma, T8700) and by measuring the intensity of immunolabeling according to a method described previously [9].

\subsection{Transcriptome Analysis}

Total RNA was isolated from $10 \mathrm{mg}$ of each tissue sample using the QIAamp RNA Mini Kit (Qiagen, Germany) immediately after sample dissection. RNA quality was monitored by electrophoresis in a denaturing agarose gel. RNA amount was determined using the fluorometer Qubit by Quant-iT RNA BR Assay Kit (Invitrogen, USA). Single-stranded DNA was synthesized using the RevertAid H Minus First Strand cDNA Synthesis Kit (Fermentas, Lithuania) using $100 \mathrm{ng}$ of total RNA, according to the manufacturer's recommendations. The quality of RNA preparations obtained was evaluated by determining the mRNA level of the housekeeping genes Actb and Gapdh using real-time PCR.

The analysis of large-scale transcriptome changes was carried out using microchip hybridization of total RNA pools. Total RNA (200 ng) from five samples of rat substantia nigra from one group was taken in each pool. Hybridization was performed on RatBasic microchips (Illumina, USA). Six independent hybridizations were carried out and the expression levels of all genes were determined for each RNA pool. The data obtained were averaged within the pool and the acquired averaged ex- 
pression profile of this pool was compared with the averaged expression profiles of other pools using the software package Genome Studio (Illumina, USA).

\subsection{Statistical Analysis}

Statistical analysis was performed using statistical software (GraphPad Prism v4.0; GraphPad Software, Inc., La Jolla, CA, USA). The control group was compared with the experimental group using Fisher's test and the statistical software STATISTICA for Windows 8.0 (StatSoft, Inc., 2007). The software package Genome Studio (Illumina, USA) and the database DAVID Bioinformatics Resources $6.7[10,11]$ were used for statistical data processing regarding the gene expression levels obtained from microchips.

Differences were considered significant when $p$ values were $<0.05$.

\section{RESULTS}

We studied a neurotoxic model of PD created via the injection of 6-OHDA into the compact part of the substantia nigra of the right brain hemisphere of rats. The analysis was carried out 2 and 4 weeks after the administration of the neurotoxin to the animals.

\subsection{6-OHDA Induced Degeneration of Dopaminergic Terminals and Motor Deficit}

We observed a significant decrease in the motor activity of the rats both at the second and the fourth weeks after the administration of 6-OHDA compared with the motor activity of the animals before the operation (Table 1).

Almost all animals developed adynamia, and collateral rotation with an intensity above $30 \mathrm{rpm}$ was observed in animals after subcutaneous administration of apomorphine. Concomitantly, no significant alterations of animal behavior were observed 1 week after drug administration.

Quantitative analysis of the intensity of immunolabeling of tyrosine hydroxylase in the caudate nucleus of

Table 1. Horizontal motor activity of the rats in the open field study.

\begin{tabular}{cc}
\hline & $\begin{array}{c}\text { The number of squares } \\
\text { crossed in three minutes }\end{array}$ \\
\hline $\begin{array}{c}\text { Before operation } \\
\text { One week after the toxin } \\
\text { administration }\end{array}$ & $68 \pm 7$ \\
Two weeks after the toxin \\
$\quad$ administration \\
$\begin{array}{c}\text { Four weeks after the toxin } \\
\text { administration }\end{array}$ \\
\hline
\end{tabular}

*These results statistically differ from the control ( $p<0.05$ (by Fisher)). both hemispheres showed a statistically significant decrease in the density of staining in the area of the caudate nucleus of the right hemisphere, which indicated a reduction of the expression of the enzyme on the side of injection of the toxin (Figures $\mathbf{1}$ and 2). Tyrosine hydroxylase expression decreased abruptly in the caudate nucleus on the side of the 6-OHDA injection by the fourth week after the microinjection of 6-OHDA into the

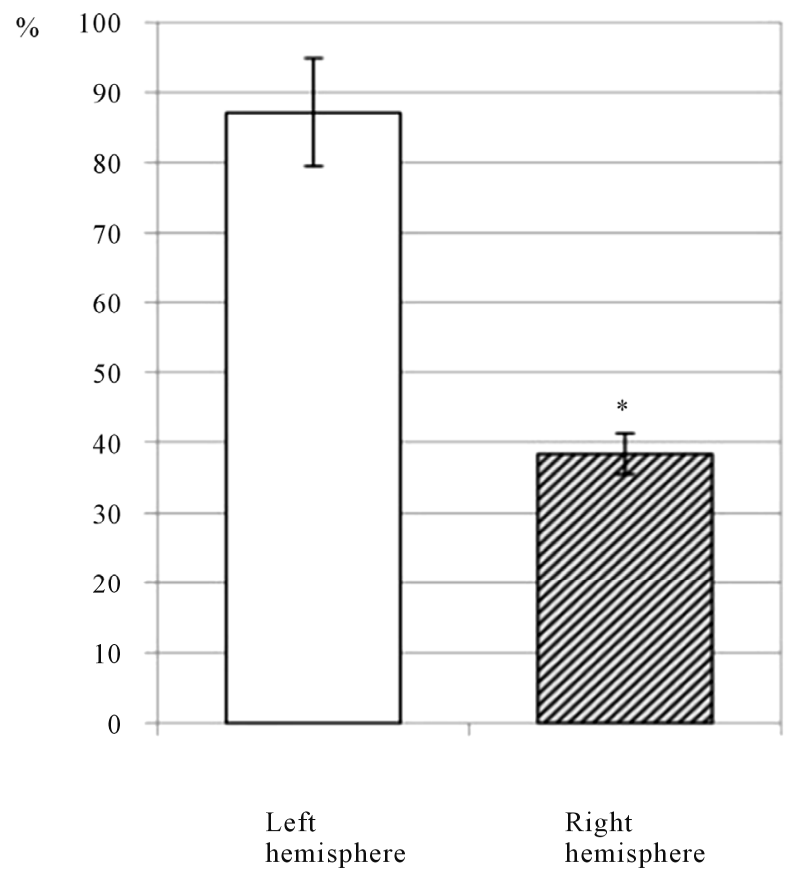

Figure 1. Changes in the intensity of immunostaining for tyrosine hydroxylase in the caudate nucleus of rats after unilateral 6-OHDA administration in the substantia nigra. Data are presented as a percentage of the left hemisphere of the native control $(100 \%)$ as $\mathrm{M} \pm \mathrm{s}$, where $\mathrm{M}-$ mean, $\mathrm{s}$ - standard deviation. $\mathrm{a}$-hemisphere into which the solvent was injected, $\mathrm{b}$ - hemisphere into which the 6-OHDA was injected. $*-\mathrm{p}<0.05$ (ANOVA, Fisher's a posteriori test).
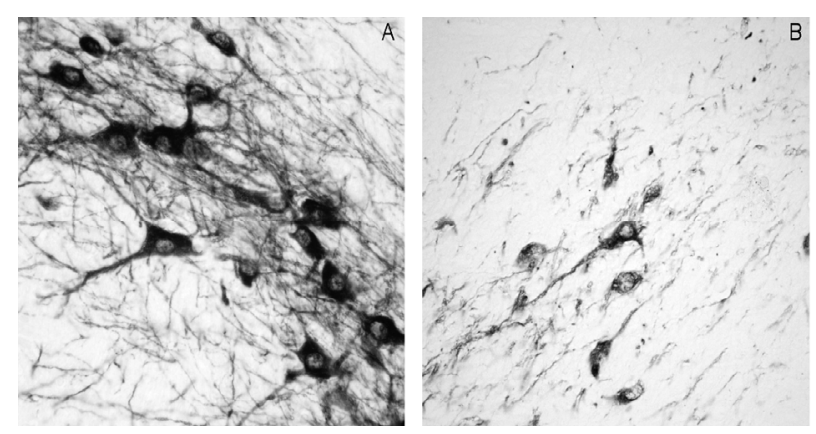

Figure 2. Immunostaining for tyrosine hydroxylase in the compact part of the substantia nigra of rats after unilateral 6OHDA administration. (A) - individual neurons in the left hemisphere into which the solvent was injected, (B) - individual neurons in the right hemisphere into which the 6-OHDA was injected. 
substantia nigra of the right hemisphere of the brain, which indicated a marked reduction in dopamine synthesis in the nigrostriatal formations, as well as the degeneration of the dopamine neurons of the substantia nigra.

\subsection{Transcripome Analysis of the Substantia Nigra}

The analysis of four rat groups (five animals in each group: two experimental and two control groups) was performed. Decapitation of the first batch of animals was carried out 2 weeks after the injection of the toxin. The second batch of animals was decapitated in 4 weeks after the injection of the toxin. Samples of the substantia nigra from each animal were taken from both cerebral hemispheres: the right, into which the toxin or vehicle was injected, and the left (native) intact side. It is essential to note that samples of the SNpc were chosen for analysis based on its remote location, away from the site of injection of the toxin or the vehicle. As a result, eight averaged sets of mRNA levels normalized to housekeeping genes were obtained.

At the first stage, data from experimental and control rat groups were compared, and the expression profiles in operated and nonoperated hemispheres of rat brains were collated. This allowed us to take into account the effects associated with the the impact of the procedure on the whole brain (for instance, the effect of postoperative stress). Consequently, 52 genes exhibited similar expression changes in the two groups (experimental and control groups). These genes were discarded from further analysis. The gene that encodes arginine vasopressin (Avp) may serve as a striking example of the genes detected at this first stage. An increase in the expression of this gene (more than 10 times) was observed. At present, it was shown that arginine vasopressin participates in the regulation of behavioral reactions, and that its upregulation may be associated with postoperative stress [12].

At the second stage, a comparison of the data obtained from the experimental and control groups of rats (which were normalized to the nonoperative hemisphere) was carried out. This comparison revealed precisely the genes that exhibited a change in expression in response to the toxic injury of neurons by 6-OHDA.

As a result, we identified 131 genes and 698 genes with significantly changed expression levels $(p<0.05)$ in the substantia nigra 2 and 4 weeks after the administration of the toxin respectively. Concomitantly, alteration in the expression of 33 genes was identified at both time points.

DAVID was used for further analysis of a panel of differentially expressed genes, which allowed us to perform a fast annotation of genes of any interest and to combine them into functional groups.

For the first time point ( 2 weeks), genes were clustered according to high significance $(\mathrm{p}<0.01$, modified Fisher extract $p$ value) into three metabolic pathways according to protein participation (Figure 3): modification of the extracellular matrix (GO:0005576, GO: 0005615, GO:0044421), signal transduction (including genes encoding signal peptides) (GO:0007165, GO: 0007218), and inflammation processes (GO:0006954).

For the second time point (4 weeks after neurotoxin injection), genes were clustered according to high significance $(\mathrm{p}<0.01$, modified Fisher extract $\mathrm{p}$ value) into five metabolic pathways (Figure 4): neuron projection (GO:0043005), normal functioning of the soma and neuron dendrites (GO:0043025, GO:0030425), synaptic transmission (GO:0007268), and transfer of nerve impulse (GO:0019226).

\begin{tabular}{|c|c|c|c|c|c|c|c|}
\hline & Annotation Cluster 1 & Enrichment Score: 4.51 & Q & & Count & P_Value & Benjamini \\
\hline 0 & SP_PIR_KEYWORDS & sianal & $\underline{\text { RI }}$ & $\bar{\square}$ & 45 & $1.5 \mathrm{E}-7$ & $4.6 \mathrm{E}-5$ \\
\hline$\square$ & UP_SEQ_FEATURE & signal peptide & RI & $\bar{\square}$ & 45 & $1.8 \mathrm{E}-7$ & $8.3 E-5$ \\
\hline 口 & SP_PIR_KEYWOROS & $\underline{\text { Secreted }}$ & $\underline{\text { RI }}$ & $\bar{\square}$ & 29 & $1.3 \mathrm{E}-6$ & $2.0 E-4$ \\
\hline$\square$ & GOTERM_BP_FAT & inflammatory response & RI & $\equiv$ & 13 & $3.1 \mathrm{E}-6$ & $4.1 E-3$ \\
\hline 0 & GOTERM_CC_FAT & extracellular space & $\underline{\text { RI }}$ & $\equiv$ & 19 & $3.9 \mathrm{E}-6$ & $8.8 E-4$ \\
\hline (1) & GOTERM_CC_FAT & extracellular recion part & $\underline{\text { RI }}$ & $\bar{\equiv}$ & 22 & $9.5 E-6$ & $7.3 E-4$ \\
\hline 0 & GOTERM_CC_FAT & extracellular recion & $\underline{\text { RT }}$ & $\bar{\square}$ & 33 & $2.3 E-5$ & $1.3 E-3$ \\
\hline$\square$ & SP_PIR_KEYWORDS & disulfide bond & $\underline{\text { RI }}$ & $\overline{\bar{\equiv}}$ & 34 & $3.7 E-4$ & $1.9 E-2$ \\
\hline 0 & SP_PIR_KEYWORDS & glycoprotein & $\underline{\mathrm{RI}}$ & $\bar{\square}$ & 44 & $5.7 E-4$ & $2.5 E-2$ \\
\hline$\square$ & GOTERM_BP_FAT & defense response & $\underline{\text { RI }}$ & $\equiv$ & 13 & $1.3 E-3$ & $6.4 E-2$ \\
\hline$\square$ & UP_SEQ_FEATURE & disulfide bond & $\underline{\text { RI }}$ & $\bar{\equiv}$ & 30 & $4.3 E-3$ & $4.8 E-1$ \\
\hline 0 & UP_SEQ_FEATURE & glycosylation site:N-linked (GICNAC....) & $\underline{\text { RI }}$ & $\bar{\square}$ & 39 & $6.6 E-3$ & $5.3 E-1$ \\
\hline
\end{tabular}

Figure 3. Functional clusterization of differentially expressed genes in the substantia nigra 2 weeks after the toxin administration. 


\begin{tabular}{|c|c|c|c|c|c|c|c|}
\hline & Annotation Cluster 1 & Enrichment Score: 5.61 & & 3 & Count & P_Value & Benjamini \\
\hline 口 & GOTERM_CC_FAT & neuron projection & $\underline{\text { RI }}$ & $\bar{\Xi}$ & 34 & $5.6 \mathrm{E}-8$ & $2.4 E-5$ \\
\hline 口 & GOTERM_CC_FAT & cell soma & $\underline{\mathrm{RI}}$ & $\overline{-}$ & 20 & $4.0 E-6$ & $8.6 E-4$ \\
\hline 0 & GOTERM_CC_FAT & cell projection & $\underline{\text { RI }}$ & $\bar{\Xi}$ & 48 & $4.4 E-6$ & $6.4 E-4$ \\
\hline 四 & GOTERM_CC_FAT & dendrite & $\underline{\text { RI }}$ & $\overline{\mathbf{a}}$ & 18 & $3.7 E-5$ & $4.0 E-3$ \\
\hline & Annotation Cluster 2 & Enrichment Score: 3.17 & (6) & 4 & Count & P_Value & Benjamini \\
\hline (0 & GOTERM_BP_FAT & transmission of nerve impulse & $\underline{\mathrm{RI}}$ & $\bar{\Xi}$ & 29 & $2.7 E-5$ & $6.5 E-2$ \\
\hline 0 & GOTERM_BP_FAT & $\underline{\text { svnaptic transmission }}$ & $\underline{\text { RI }}$ & E & 26 & $3.3 E-5$ & $4.0 E-2$ \\
\hline 四 & GOTERM_BP_FAT & cell-cell sianaling & $\underline{\mathrm{RT}}$ & $\bar{\Xi}$ & 37 & $7.5 E-4$ & $2.4 E-1$ \\
\hline$\square$ & GOTERM_BP_FAT & neurological system process & $\underline{\mathrm{RT}}$ & $\equiv$ & 46 & $2.9 \mathrm{E}-1$ & $9.4 E-1$ \\
\hline
\end{tabular}

Figure 4. Functional clusterization of differentially expressed genes in the substantia nigra 4 weeks after the toxin administration.

Among all the metabolic processes and genes, identified here, we chose genes associated with neuron projection for a more detailed analysis (Table 2), because this metabolic process received the highest rating of statistical significance during the cluster analysis $(\mathrm{p}=0.000024)$. As can be seen from the data presented in Table 2, the decrease in the relative mRNA levels of genes associated with neuron projection in the substantia nigra of the brain of rats exposed to the neurotoxin compared with the control group of animals was mainly observed. An increase in relative mRNA levels of several genes was detected in the substantia nigra of the brain of rats exposed to the neurotoxin compared with the control group of animals; these genes are marked in gray in Table 2.

\section{DISCUSSION}

One of the approaches that can be used to study the pathogenesis of PD and identify new candidate genes involved in the disease is the development of a model of the disease and its subsequent analysis. We used a model based on the toxic damage of dopaminergic neurons of the substantia nigra caused by the local injection of 6OHDA into the brains of the rats (directly into the SNpc), to create a Parkinson-like phenotype. In this case, the experimental animals developed pronounced changes in behavior that were typical of a Parkinson-like phenotype only in 2 weeks after the administration of the toxin. In this regard, we studied transcriptome alterations in the substantia nigra of the brains of rats in 2 weeks after toxin administration, the time point at which the rats developed the Parkinson-like phenotype, and in 4 weeks after toxin administration, the time point at which maximal changes in the behavior of animals were observed.

The transcriptome analysis of the substantia nigra of the brains of rats at the first time point (2 weeks) revealed changes in the metabolic processes involved, occurring first in cell matrix formation. This likely indi- cates, that during this time nonspecific effects associated with the response to surgery took place in the substantia nigra of the rats. Specific effects were either absent or expressed very poorly that did not allow their detection during clustering. Concomitantly, the important role of changes in cell-cell interactions and of the structure of the extracellular matrix in the initiation of the death of dopaminergic neurons cannot be excluded. The identification of protein-coding genes involved in inflammation can be considered the only relatively specific effect. It has been shown previously that inflammation may be involved in the pathogenesis of PD. It was established that the activation of microglia takes place in the substantia nigra of patients with $\mathrm{PD}$, and that this process precedes, and may initiate, neuronal death [13]. Activated microglia can produce various anti-inflammatory cytokines. This is accompanied by the activation of the expression of the gene that encodes neuronal nitric oxide synthase (nNOS), which in turn results in the increase in the levels of nitric oxide and the enhancement of oxidative stress. This increases the sensitivity of dopaminergic neurons to oxidative stress and the probability of their death. Similar effects were observed in the early stages in the study of the 6-OHDA model created via the introduction of the neurotoxin into the striatum. Transcriptome analysis identified pathways associated with cell adhesion and immune responses 3, 7, and 14 days after the injection of the toxin into the substantia nigra of rat brains [14].

Concomitantly, the situation changed dramatically and a response associated with damage to the nervous tissue was observed 4 weeks after neurotoxin administration. This was supported by a cluster analysis of the genes that were differentially expressed at this time point. As a result, we identified five metabolic pathways containing predominantly genes, that encode protein products that are involved in the processes of neuron projection, normal functioning of the soma and dendrites of neurons, 
Table 2. List of genes of neuron projection and their relative expression levels in the substantia nigra 2 and 4 weeks after the toxin administration (Gene expression level in the substantia nigra of the control group of the rats was accepted as 1.0 , only significant ( $\mathrm{p}<$ 0.05 ) changes of expression level are given).

\begin{tabular}{|c|c|c|c|}
\hline GENE & Protein & 2 weeks & Four weeks \\
\hline WFS1 & Wolfram syndrome 1 (wolframin) & - & 0.63 \\
\hline ACTN2 & Actinin, alpha 2 & - & 0.75 \\
\hline$A R C$ & Activity-regulated cytoskeleton-associated protein & 1.87 & 0.65 \\
\hline$B A S P 1$ & Brain abundant, membrane attached signal protein 1 & - & 0.68 \\
\hline CTNNA2 & Catenin (cadherin-associated protein), alpha 2 & - & 1.31 \\
\hline CLCN2 & Chloride channel 2 & - & 0.61 \\
\hline CCK & Cholecystokinin & - & 0.51 \\
\hline CHRNA4 & Cholinergic receptor, nicotinic, alpha 4 & - & 1.99 \\
\hline CHRNA5 & Cholinergic receptor, nicotinic, alpha 5 & - & 0.51 \\
\hline CDK5 & cyclin-dependent kinase 5 & - & 0.74 \\
\hline$D D N$ & dendrin & - & 0.80 \\
\hline$D V L 1$ & dishevelled, dsh homolog 1 (Drosophila); dishevelled, dsh homolog 1 (Drosophila)-like 1 & - & 0.55 \\
\hline$E R M N$ & Ermin, ERM-like protein & - & 1.26 \\
\hline GABBR2 & gamma-aminobutyric acid (GABA) B receptor & - & 0.79 \\
\hline$G A D 1$ & glutamate decarboxylase 1 (brain, $67 \mathrm{kDa}$ ) & - & 1.39 \\
\hline GRIN1 & Glutamate receptor, ionotropic, N-methyl D-aspartate 1 & - & 0.72 \\
\hline GRM1 & Glutamate receptor 1 & - & 1.62 \\
\hline GOT1 & Glutamic-oxaloacetic transaminase 1 soluble (aspartate aminotransferase 1) & - & 1.32 \\
\hline KALRN & kalirin, RhoGEF kinase & - & 0.59 \\
\hline KPTN & kaptin (actin binding protein) & - & 0.63 \\
\hline$M A G E E 1$ & melanoma antigen family E & - & 1.27 \\
\hline MAP1S & Microtubule-associated protein 1S & - & 0.72 \\
\hline MAP2 & Microtubule-associated protein 2 & - & 0.44 \\
\hline MAPK8IP1 & Mitogen-activated protein kinase 8 interacting protein 1 & - & 0.77 \\
\hline MAPK8IP3 & Mitogen-activated protein kinase 8 interacting protein 3 & - & 0.73 \\
\hline NOV & Nephroblastoma overexpressed gene & - & 0.47 \\
\hline$N M U$ & Neuromedin U & - & 2.92 \\
\hline NGEF & Neuronal guanine nucleotide exchange factor & - & 0.71 \\
\hline$P P T 1$ & Palmitoyl-protein thioesterase 1 & - & 1.27 \\
\hline$P V A L B$ & Parvalbumin & - & 2.57 \\
\hline SCN1 A & Sodium channel, voltage-gated, type I, alpha subunit & 0.61 & 1.76 \\
\hline TAC1 & Tachykinin, precursor 1 & - & 0.65 \\
\hline TSC2 & Tuberous sclerosis 2 & - & 0.71 \\
\hline CNTF & $\begin{array}{c}\text { CNTF zinc finger protein } 91 \text { homolog (mouse); ZFP91-CNTF readthrough transcript; } \\
\text { ciliary neurotrophic factor }\end{array}$ & - & 1.52 \\
\hline
\end{tabular}


synaptic transmission, and transmission of nerve impulses.

In this case, the strong nonspecific effects observed 2 weeks after the administration of 6-OHDA, were not discovered at this later time point. Thus, a change of a predominantly nonspecific reaction of the nervous tissue to a more specific response occured.

Among all the metabolic processes and genes identified in the analysis, we reviewed in more detail those genes that are involved in neuron projection. In this case, we observed mainly a decrease in the relative mRNA levels of genes associated with neuron projection in the substantia nigra of the brain of the rats exposed to the neurotoxin compared with control animals (Table 2). On the one hand, the changes observed may be the result of massive loss of neurons in the brain areas studied 4 weeks after the administration of 6-OHDA. On the other hand, the decrease in the expression of these genes can lead to the development of the degradation processes of the neurons. An increase in the relative levels of mRNA of several genes was shown in the substantia nigra of the brain of rats exposed to the neurotoxin compared with control animals. The activation of the expression of these genes may be associated with a protective response of neurons to the action of the toxin and processes of neurodegeneration. To date, and according to the data available, most of these genes are not involved in the processes that can lead to the degradation of dopaminergic neurons. For example, the greatest increase in expression (by almost three times) (Table 2) was observed for the neuromedin gene (NMU), which is a neuropeptide. However, the precise function of this protein remains unknown. It may play an important role in energy metabolism, as it is involved in the processes of regulation of appetite. There is also evidence that this gene is involved in the pathogenesis of some cancers, such as lung cancer, bladder cancer, and myeloid leukemia [15]. We cannot exclude its participation in the pathogenesis of $\mathrm{PD}$, as many of the functions of this protein remain unknown, and we were able to show a significant increase in its mRNA in the substantia nigra of rats with a Parkinson-like phenotype.

We can assume that only three genes (PPT1, GRM1, and PVALB), the expression of which was increased, are involved in processes leading to neurodegeneration in PD. The PPT1 gene, for instance, encodes the palmitoylated protein thioesterase 1 , which participates directly in lysosomal protein degradation [16]. Interestingly, it has been shown that disturbance of the lysosomal degradation of proteins may be involved in the pathogenesis of PD [17].

Moreover, the GRM1 gene encodes a glutamate receptor, which is one of the participants in the glutamatergic system. This gene plays an important role in the syn- aptic plasticity and may be involved in the development of cerebellar ataxia [18]. The PVALB gene encodes parvalbumin, which has a high affinity for calcium-binding proteins, such as calmodulin. This similarity suggests its important role in calcium homeostasis. The disruption of calcium homeostasis and its accumulation in cells can cause the development of oxidative stress and mitochondrial dysfunction, which are the main processes that lead to the death of dopaminergic neurons [19]. In addition, the immunohistochemical analysis of the substantia nigra from the autopsy material from patients with PD showed an increase in the amount of parvalbumin in dopaminergic neurons [20]. We also found a significant (2.57 times) increase in the level of the PVALB mRNA in the substantia nigra of the brain of rats treated with the neurotoxin.

In conclusion, the transcriptome analysis performed in a rat model of PD generated via the injection of 6-OHDA showed that most genes develop a nonspecific response that was associated primarily with the introduction of the toxin directly into the brain. This model is probably not completely adequate to study changes in gene expression. It is possible that future studies of other neurotoxic models, especially MPTP models, which reproduce presymptomatic and early symptomatic stages of PD [21], will allow the identification of genes and processes that are involved in the pathogenesis of the early stages of PD, and the investigation of compensatory mechanisms that will ensure long-term asymptomatic disease.

\section{ACKNOWLEDGEMENTS}

This work was supported by the Russian Foundation for Basic Research (projects no. 12-04-31091, 12-04-01183-a), as well as by programs of the Russian Academy of Sciences (Molecular and Cellular Biology, Fundamental Sciences for Medicine), state contracts (no. 16.740.11.0630, 8605, and 8851).

\section{REFERENCES}

[1] Nussbaum, R.L. and Polymeropoulos, M.H. (1997) Genetics of Parkinson's disease. Human Molecular Genetics, 6, 1687-1691. doi:10.1093/hmg/6.10.1687

[2] Shadrina, M.I., Slominsky, P.A. and Limborska, S.A. (2010) Molecular mechanisms of pathogenesis of Parkinson's disease. International Review of Cell \& Molecular Biology, 281, 229-266. doi:10.1016/S1937-6448(10)81006-8

[3] Saiki, S., Sato, S. and Hattori, N. (2012) Molecular pathogenesis of Parkinson's disease: Update. Journal of Neurology, Neurosurgery \& Psychiatry, 83, 430-436. doi:10.1136/jnnp-2011-301205

[4] Blandini, F. and Armentero, M.T. (2012) Animal models of Parkinson's disease. FEBS Journal, 279, 1156-1166. doi:10.1111/j.1742-4658.2012.08491.x 
[5] Ungerstedt, U., Ljungberg, T. and Steg, G. (1974) Behavioral, physiological, and neurochemical changes after 6-hydroxydopamine-induced degeneration of the nigrostriatal dopamine neurons. Advances in Neurology, 5, 421-426.

[6] Sauer, H. and Oertel, W.H. (1994) Progressive degeneration of nigrostriatal dopamine neurons following intrastriatal terminal lesions with 6-hydroxydopamine: A combined retrograde tracing and immunocytochemical study in the rat. Neuroscience, 59, 401-415. doi:10.1016/0306-4522(94)90605-X

[7] Blandini, F., Armentero, M.T. and Martignoni, E. (2008) The 6-hydroxydopamine model: News from the past. Parkinsonism \& Related Disorders, 14, S124-129. doi:10.1016/j.parkreldis.2008.04.015

[8] Chen, X.Y., Li, J., Qi, W.Q. and Shen, S.H. (2007) Experimental change on dopaminergic neurons in striatum of Parkinson disease rats. Histology and Histopathology, 22, 1085-1090.

[9] Urbanavicius, J., Ferreira, M., Costa, G., Abin-Carriquiry, J.A., Wonnacott, S., et al. (2007) Nicotine induces tyrosine hydroxylase plasticity in the neurodegenerating striatum. Journal of Neurochemistry, 102, 723-730. doi:10.1111/j.1471-4159.2007.04560.x

[10] Huang da, W., Sherman, B.T., and Lempicki, R.A. (2009) Bioinformatics enrichment tools: Paths toward the comprehensive functional analysis of large gene lists. Nucleic Acids Research, 37, 1-13. doi:10.1093/nar/gkn923

[11] Huang da, W., Sherman, B.T. and Lempicki, R.A. (2009) Systematic and integrative analysis of large gene lists using DAVID bioinformatics resources. Nature Protocols, 4, 44-57. doi:10.1038/nprot.2008.211

[12] Caldwell, H.K., Lee, H.J., Macbeth, A.H. and Young, W.S. (2008) Vasopressin: Behavioral roles of an "original" neuropeptide. Progress in Neurobiology, 84, 1-24. doi:10.1016/j.pneurobio.2007.10.007

[13] Orr, C.F., Rowe, D.B. and Halliday, G.M. (2002) An inflammatory review of Parkinson's disease. Progress in Neurobiology, 68, 325-340.
doi:10.1016/S0301-0082(02)00127-2

[14] Na, S.J., DiLella, A.G., Lis, E.V., Jones, K., Levine, D.M., et al. (2010) Molecular profiling of a 6-hydroxydopamine model of Parkinson's disease. Neurochemical Research, 35, 761-772. doi:10.1007/s11064-010-0133-3

[15] Mitchell, J.D., Maguire, J.J. and Davenport, A.P. (2009) Emerging pharmacology and physiology of neuromedin $\mathrm{U}$ and the structurally related peptide neuromedin $\mathrm{S}$. British Journal of Pharmacology, 158, 87-103. doi:10.1111/j.1476-5381.2009.00252.x

[16] Ohno, K., Saito, S., Sugawara, K., Suzuki, T., Togawa, T., et al. (2009) Structural basis of neuronal ceroid lipofuscinosis 1. Brain \& Development, 32, 524-530. doi:10.1016/j.braindev.2009.08.010

[17] Zhang, L., Sheng, R. and Qin, Z. (2009) The lysosome and neurodegenerative diseases. Acta Biochimica et Biophysica Sinica, 41, 437-445. doi:10.1093/abbs/gmp031

[18] Guergueltcheva, V., Azmanov, D.N., Angelicheva, D., Smith, K.R., Chamova, T., et al. (2012) Autosomal-recessive congenital cerebellar ataxia is caused by mutations in metabotropic glutamate receptor 1. The American Journal of Human Genetics, 91, 553-564. doi:10.1016/j.ajhg.2012.07.019

[19] Imai, Y. and Lu, B. (2011) Mitochondrial dynamics and mitophagy in Parkinson's disease: Disordered cellular power plant becomes a big deal in a major movement disorder. Current Opinion in Neurobiology, 21, 935-941. doi:10.1016/j.conb.2011.10.016

[20] Soos, J., Engelhardt, J.I., Siklos, L., Havas, L. and Majtenyi, K. (2004) The expression of PARP, NF-kappa B and parvalbumin is increased in Parkinson disease. Neuroreport, 15, 1715-1718. doi:10.1097/01.wnr.0000136175.51954.ce

[21] Ugrumov, M.V., Khaindrava, V.G., Kozina, E.A., Kucheryanu, V.G., Bocharov, E.V., et al. (2011) Modeling of presymptomatic and symptomatic stages of parkinsonism in mice. Neuroscience, 181, 175-188. doi:10.1016/j.neuroscience.2011.03.007 